\title{
Nueva propuesta teórica sobre obstáculos epistemológicos de aprendizaje. El caso del metabolismo de los carbohidratos
}

\section{New theoretical proposal on epistemological learning obstacles. The case of carbohydrate metabolism}

Sofía J. Garófalo, Manuel Alonso

Departamento de Ciencias Biológicas, Ciclo Básico Común,

Universidad de Buenos Aires, Ramos Mejia 841, C1405CAE, Ciudad de Buenos Aires, Argentina

sjgarofalo@gmail.com, m_alonso@live.com.ar

Lydia R. Galagovsky

Facultad de Ciencias Exactas y Naturales, Instituto Centro de Formación e Investigación en Enseñanza de las Ciencias (CEFIEC),

Universidad de Buenos Aires, Ramos Mejía 841, C1405CAE, Ciudad de Buenos Aires, Argentina

lyrgala@qo.fcen.uba.ar

RESUMEN • La utilización de tres modelos científicos definidos ad hoc sobre el metabolismo de los hidratos de carbono permitió analizar numerosos errores detectados en el aprendizaje de estudiantes universitarios. Este análisis llevó a considerar la existencia de dos clases diferentes de obstáculos epistemológicos subyacentes al aprendizaje (OEA): los de tipo brecha y los de tipo puente. Los primeros se manifiestan por la falta de modelos mentales en los que los estudiantes puedan anclar o engarzar sus aprendizajes; y los segundos se corresponderían a ideas consolidadas para el sujeto que actuarían como modelos mentales idiosincrásicos, funcionales pero erróneos desde el punto de vista científico. Este aporte teórico, basado en evidencias empíricas, constituye una alternativa de análisis que va más allá de la simple enumeración de los errores de los estudiantes.

PALABRAS CLAVE: obstáculos epistemológicos de aprendizaje; metabolismo; hidratos de carbono.

ABSTRACT - The use of three ad hoc scientific models on the metabolism of carbohydrates can consider the existence of underlying epistemological obstacles that lead to university students to make a lot of mistakes. This approach led to interpret students' failures into two different epistemological learning obstacles (ELO): «Gap Type» and «Bridge Type». The first one ELO corresponds to cognitive obstacles arising from the lack of knowledge; this means the lack of mental models. The second one corresponds to consolidated previous ideas that act as alternative underlying mental models. This theoretical contribution, based on evidences, allows analysis that goes beyond the simple listing of students' misunderstandings.

KEYWORDS: learning difficulties; epistemological obstacles; metabolism; carbohydrates.

Fecha de recepción: octubre 2012 • Aceptado: septiembre 2013

Garófalo, S. J., Alonso, M., Galagovsky, L. (2014) Nueva propuesta teórica sobre obstáculos epistemológicos de aprendizaje. El caso del metabolismo de los carbohidratos. 


\section{INTRODUCCIÓN}

Los hidratos de carbono y su metabolismo constituyen uno de los temas más importantes enseñados reiteradamente en el nivel secundario y, muy especialmente, en el nivel universitario en carreras relacionadas con la biología, la química, la medicina y la nutrición.

El tema "Metabolismo de los Hidratos de Carbono» (MHC) resulta estructurante (Gagliardi, 1986) para la interpretación e integración de conocimientos bioquímicos, celulares y fisiológicos en los seres vivos. Su enseñanza involucra el estudio de variadas estructuras moleculares y de sus reacciones químicas, el papel de los enzimas específicos involucrados, de los mecanismos de transporte a través de la membrana, la captación específica de tejido y las regulaciones de vías metabólicas como la glucólisis, glucogenogénesis, glucogenólisis, gluconeogénesis o de las pentosas (Campbell y Reece, 2005; Berg et al., 2007).

Numerosas investigaciones han demostrado graves dificultades para la comprensión del MHC, tanto para estudiantes de secundaria como universitarios. Por ejemplo, Songer y Mintzes (1994) han mostrado problemas de aprendizaje del tema en estudiantes de Biología (nivel College, de Estados Unidos). Banet Hernández (2001) detalló un amplio panorama de las distintas dificultades que presentan estudiantes de escuela media española respecto de la nutrición, sus aspectos fisiológicos y bioquímicos, y sobre el proceso de respiración celular.

En investigaciones recientes (Oliveira et al., 2003; Luz, 2008; Luz et al., 2008) se detectaron fallos en el aprendizaje del MHC en estudiantes y docentes de ocho carreras universitarias -de las áreas biomédica, humanística y tecnológicas- de seis universidades públicas de Río de Janeiro (Brasil). Asimismo, Cliff (2006), en un estudio realizado en estudiantes de la Universidad de Niágara (Estados Unidos), ha señalado que, a pesar del uso de estrategias de enseñanza específicas, son difíciles de erradicar las concepciones erróneas acerca de los procesos fisiológicos de respiración celular.

La preocupación de educadores de niveles universitarios ha originado propuestas didácticas para la enseńanza del MHC. Por ejemplo, se ha sugerido trabajar sobre dietas con diversos contenidos en hidratos de carbono (Pogozelski et al., 2005); o detectar en sangre -de estudiantes voluntarios- valores de glucosa y triacilgliceroles, antes y después de comer pizza o pasta (Passos et al., 2006), o realizar juegos de rol para proporcionar a los estudiantes una visión macroscópica y animada de los procesos de glucólisis y del ciclo de Krebs (Ross et al., 2008). En ninguna de estas publicaciones se analizaron resultados de aprendizaje, pues el énfasis estuvo puesto en la motivación que generaban tales propuestas didácticas.

En el presente trabajo se han investigado errores de aprendizaje del tema MHC en estudiantes universitarios de la asignatura Biología - pertenecientes al primer año de diversas carreras de la Universidad de Buenos Aires- en función de fallos en la construcción de tres modelos que estructurarían conceptualmente el tema: el Modelo Fisiológico del Ciclo del Carbono en los Heterótrofos (MFCCHet); el Modelo Fisiológico de la Disponibilidad de Metabolitos (MFDMet), y el Modelo Fisiológico de la Captación y Homeostasis de la Glucosa en Sangre (MFCHGSang). Los resultados llevaron a proponer la existencia de dos tipos de obstáculos epistemológicos subyacentes al aprendizaje del MHC: los de tipo brecha y los de tipo puente. Esta nueva propuesta teórica sobre obstáculos podrá servir como base para futuras investigaciones sobre fallos en el aprendizaje de otras temáticas.

\section{MODELOS CIENTÍFICOS Y MODELOS MENTALES}

El término modelo presenta carácter polisémico (Moreira et al.; 2002; Justi, 2006; Adúriz-Bravo, 2010; Lombardi, 2010). Estany (1993) señala: «... hay una norma clara referente a la utilización del término modelo, a saber, no utilizarla nunca sin antes delimitar en qué sentido se utiliza». El presente trabajo 
no pretende hacer una revisión de la amplia variedad de significados y sentidos de dicho término, pero requiere distinguir cuatro categorías diferentes:

El «modelo científico explícito» es un recorte y secuenciación de contenidos complejos e imbricados que remiten a concepciones eruditas presentes en textos o discursos tales como publicaciones, libros de textos, exposiciones de clase, etc. (Galagovsky et al., 2009; Garófalo et al., 2013). Estos modelos científicos explícitos se expresan mediante diversos lenguajes -verbal, gráfico, de fórmulas, matemático, etc.-, que se complementan y retroalimentan para conformar explicaciones temáticas. Así, estos modelos científicos explícitos están constituidos por elementos informativos, secuencialmente organizados, que son la interfase necesaria para establecer la comunicación entre los sujetos expertos y los novatos. Para el MHC se reconocen en la literatura numerosos modelos científicos explícitos, entre los que se destacan los procesos de glucólisis, ciclo de Krebs y respiración celular, que se encuentran en la bibliografía de referencia de la asignatura Biología y que habían sido enseñados y tomados en la evaluación parcial de los estudiantes de la muestra analizada en este trabajo.

Por otro lado, resulta interesante la definición de modelo mental como un mecanismo del pensamiento mediante el cual un ser humano intenta explicar cómo funciona el mundo real (Gutiérrez, 2005; Johnson Laird, 2005). Los sujetos expertos habrían construido sus «modelos mentales expertos» a lo largo de su formación profesional específica, tras años de estudio y apropiación de los modelos aceptados por la comunidad científica. Claramente, estos modelos mentales difieren de aquellos que construyen los estudiantes novatos a partir de recibir la enseñanza secuencial de partes de los modelos científicos explícitos; los estudiantes novatos construyen en sus mentes «modelos mentales idiosincrásicos» (Galagovsky et al., 2009; Garófalo et al., 2013).

Finalmente, en este trabajo definimos «modelos científicos ad hoc» a tres entramados particulares de modelos científicos explícitos sobre el MHC que permiten articular ideas químicas, bioquímicas y fisiológicas relevantes sobre el tema. Es decir, indagar sobre estos modelos científicos ad hoc permitiría detectar si el aprendizaje de diferentes modelos científicos explícitos separados impediría a los estudiantes construir en sus mentes estos modelos integradores.

\section{Tres modelos científicos ad hoc integradores del tema metabolismo de hidratos de carbono}

En las clases universitarias observadas, se presentó gran cantidad de información sobre el MHC, que se corresponde con el listado de temas de textos, como el de Alberts et al. (2002), Campbell y Reece (2005) y Curtis et al. (2008). Esta información incluyó los tradicionales modelos científicos explícitos fundamentales del tema, y los estudiantes fueron posteriormente evaluados en un examen parcial en el que se solicitaban respuestas de tipo semejante a las que habían sido presentadas durante la enseńanza.

En el presente trabajo, la compleja temática del MHC fue organizada en torno a tres modelos científicos ad hoc cuyas características fundamentales dieran cuenta de enfoques fisiológicos y químicos subyacentes a cuestiones biológicas. Es decir, estos tres modelos constituyen enlaces entre enfoques bioquímicos y aquellos otros provenientes de la biología celular, que permiten en conjunto interpretar los fenómenos fisiológicos macroscópicos implicados en el MHC. Los modelos propuestos son los siguientes: ${ }^{1}$

Modelo Fisiológico del Ciclo del Carbono en los Heterótrofos (MFCCHet)

Modelo Fisiológico de la Disponibilidad de Metabolitos (MFDMet)

Modelo Fisiológico de la Captación y Homeostasis de la Glucosa en Sangre (MFCHGSang)

1. Esta propuesta es original, no tiene antecedentes bibliográficos. 


\section{Conceptos principales de cada modelo:}

Modelo Fisiológico del Ciclo del Carbono en los Heterótrofos (MFCCHet). Supone la comprensión de procesos de óxido-reducción de compuestos de carbono pertenecientes a especies químicas que se encuentran tanto en la atmósfera como en los organismos vivos. Estos procesos químicos son la forma en que la mayoría de los seres vivos extraen del entorno la energía que les permite su supervivencia.

El ciclo incluye los procesos fotosintéticos de los autótrofos que transforman el $\mathrm{CO}_{2}$ atmosférico (especie totalmente oxidada del carbono) en compuestos reducidos de carbono como, por ejemplo, los carbohidratos. A su vez, las células de autótrofos y heterótrofos, mediante la respiración celular, reoxidan, controladamente, compuestos reducidos de carbono a dióxido de carbono. De esta forma, se libera la energía necesaria para sus procesos vitales, cerrándose el ciclo con la consiguiente eliminación de $\mathrm{CO}_{2}$ a la atmósfera.

Modelo Fisiológico de la disponibilidad de Metabolitos (MFDMet). Supone la comprensión de que las biomoléculas se construyen a partir de una serie pequeña de precursores (compuestos químicos de pocos carbonos) que permiten conectar entre sí las vías catabólicas y anabólicas.

Es frecuente que la enseñanza del MHC haga hincapié en la obtención de energía proveniente de vías catabólicas como la glucolisis y la respiración celular (Luz, 2008). Sin embargo, el estudio de procesos degradativos y biosintéticos (como los incluidos en el MHC) debería comprenderse tanto desde el punto de vista del requerimiento energético como de las estructuras moleculares, que constituyen la materia involucrada en dichos procesos. Entender la interconexión de las vías anabólicas y catabólicas significa apreciar que la disponibilidad de metabolitos compartidos juega un rol fundamental, y que esta depende, entre otros factores, del estado nutricional del individuo.

Modelo Fisiológico de la Captación y Homeostasis de la Glucosa en Sangre (MFCHGSang). Supone comprender cómo el organismo capta e incorpora la glucosa de los alimentos, y cómo la glucosa se distribuye homogéneamente en la sangre pero es captada en forma diferencial por las células de los distintos tejidos por mecanismos de transporte específicos. A su vez, requiere la comprensión del carácter homogéneo pero fluctuante de la glucemia mediada por la acción de las hormonas reguladoras de esta concentración. Entender estos procesos ha demandando a los científicos la elaboración de los conceptos de afinidad para los enzimas y sus correspondientes sustratos, asociada a los valores fisicoquímicos de $\mathrm{K}_{\mathrm{M}}$ (contante de Michaelis-Menten) y de $\mathrm{V}_{\max }$ (velocidad enzimática máxima), y las relaciones de estos parámetros generales con los transportadores y enzimas específicos en el contexto de MHC.

La propuesta de estos tres modelos ad hoc ha requerido una revisión histórica de los conceptos involucrados, verificándose que sus construcciones demandaron mucho tiempo, hasta que se propusieron y acordaron respuestas a las ingeniosas preguntas y originales experimentos, que guiaron la actividad de científicos de renombre. Así, el MFCCHet fue construido a lo largo de varios siglos, a partir de preguntas iniciales tales como de dónde obtienen las plantas la materia que genera su aumento de tamaño -planteadas por van Helmont, en el siglo XVII-, y qué relación existe entre la combustión y la respiración -planteadas por Priestley, Lavoisier y Laplace en el siglo XVIII- (Hudson, 1992; Garófalo, 2010). Más cercanas a nuestros días, en la década de 1940, las investigaciones de Calvin sobre la fotosíntesis permitieron detectar la secuencia de reacciones químicas generadas por las plantas al transformar dióxido de carbono gaseoso y agua en hidratos de carbono y oxígeno, proceso que en la actualidad se conoce como ciclo de Calvin (Palmer, 2001).

Por su parte, el MFDMet implicó conocer que los destinos metabólicos de la glucosa involucran el momento nutricional por el que atraviesa un organismo. Así, Bernard, en el siglo XIX, esclareció que las sustancias resultantes de las acciones químicas sobre los alimentos son absorbidas en el intestino, y que el medio interno de un organismo se mantiene constante a pesar de los cambios que ocurran en su entorno. Kasahara y Hinkle, en 1977, se preguntaron cómo entran los nutrientes -como la 
glucosa- en las células, y descubrieron el primer transportador de glucosa, aislado de membranas de eritrocitos (Díaz Hernández y Burgos Herrera, 2002). Buchner, a finales del siglo XIX, se planteó si las reacciones de fermentación se pueden llevar a cabo sin células, y denominó enzima a la sustancia que causa tal proceso. Embden y Meyerhorf, en las primeras décadas del siglo XX, dilucidaron cuál es la ruta metabólica que involucra la oxidación de la glucosa; mientras que Krebs, para esa misma época, logró responder magistralmente cómo concluye la oxidación de la glucosa generando el $\mathrm{CO}_{2}$ como producto de la respiración celular, al proponer el ciclo de los ácidos tricarboxílicos, conocido también por el nombre de este investigador (Lehninger, 1978).

Finalmente, el MFCHGSang se basa en la comprensión de los mecanismos de transporte a través de membrana y la distribución homogénea de glucosa en sangre, aunque fluctuante según las condiciones nutricionales del individuo. Bernard, en el siglo XIX, aisló glucógeno del hígado, mostrando que se convertía en la glucosa sanguínea, y también descubrió el proceso de gluconeogénesis (Lehninger, 1978) y, de esta forma, respondió al interrogante que plantea la existencia de un suministro interno de glucosa cuando esta no se ingiere. Por su parte, Banting et al. (1922), al descubrir la insulina, empezaron a esclarecer cómo se regula tal suministro de glucosa.

A su vez, las investigaciones de Sols García y Crane, en 1947, acerca de la hexoquinasa, sentaron las bases que posteriormente permitirían comprender cómo se explica la captación diferencial de la glucosa, en las células de los distintos tejidos. Además Sols, con sus trabajos sobre la fosfotructoquinasa, contribuyó a comprender cómo se regula la oxidación de la glucosa de acuerdo a las condiciones energéticas de la célula (Teixidó Gómez, 2003).

\section{METODOLOGÍA}

A partir de la consideración de los tres modelos científicos ad hoc, se diseñaron dos instrumentos de indagación que permitieran explorar si los estudiantes habrían construido adecuadamente los respectivos modelos mentales. Estos instrumentos -una entrevista y un cuestionario semiestructurado- no tenían una correspondencia unívoca con cada uno de los tres modelos científicos; no obstante, las respuestas darían cuenta del grado de comprensión de tales modelos.

Los instrumentos de indagación fueron aplicados a estudiantes voluntarios de dos cohortes secuenciales de la materia cuatrimestral Biología del primer año de la Universidad de Buenos Aires, ${ }^{2}$ una vez que fuera impartida la enseñanza completa del tema MHC y que los estudiantes hubieran sido evaluados en las instancias tradicionales (exámenes parciales).

El estudio no buscaba indagar el razonamiento de cada sujeto individual; por consiguiente, las respuestas se analizaron como totalidades sobre el conjunto de la población.

\section{La entrevista}

La entrevista consistió en un problema abierto y tres preguntas subsiguientes (Ericsson y Simon, 1999), que se muestran en los cuadros 1 y 2 .

2. La investigación es parte de un trabajo de tesis doctoral que abarcó a un total de 800 estudiantes de cuatro asignaturas de sendas carreras universitarias (Garófalo, 2010). 
Cuadro 1.

Problema abierto de la entrevista

Una persona ingiere una porción de pizza (consideraremos el almidón como componente principal). ¿̇xiste la posibilidad de encontrar en la atmósfera, en algún momento, alguno de los átomos de carbono que forman parte de la glucosa del almidón de esa pizza? Menciona cómo y qué vías metabólicas estarían involucradas.

Cuadro 2.

Tres preguntas de la entrevista

1. ¿Sabes de dónde proviene la glucosa involucrada en la respiración celular?

2. ¿Cómo y por dónde piensas que se incorpora la glucosa a la sangre?

3. ¿Qué sucede con la glucosa una vez en sangre?

La contestación afirmativa al problema abierto daría cuenta de estudiantes que hubieran comprendido el MFCCHet, y dieron argumentaciones que fueron registradas, analizadas y cuantificadas. ${ }^{3}$

Por su parte, independientemente de la respuesta afirmativa o negativa al problema abierto, todo entrevistado debió responder las tres preguntas del cuadro 2. Estas se referían a los contextos de significación subyacentes a las respuestas al problema abierto (Ericsson y Simon, 1999). De esta forma, se podían indagar los modelos mentales idiosincrásicos que habrían construido los estudiantes. Las preguntas 1 y 3 correctamente respondidas darían cuenta de que habrían comprendido el MFDMet, y respuestas correctas a las preguntas 2 y 3 mostrarían la comprensión de elementos de MFCHGSang.

\section{El cuestionario semiestructurado}

Dado que las respuestas a las entrevistas pusieron en evidencia errores conceptuales en muchos estudiantes, resultó apropiado triangular los resultados. Para ello, tres semanas más tarde se aplicó un nuevo instrumento: un cuestionario semiestructurado (cuadro 3) con algunas preguntas abiertas y otras del tipo de opción múltiple.

Las entrevistas fueron resueltas por 182 estudiantes voluntarios. En cambio, dado que el cuestionario semiestructurado fue diferido, la población voluntaria resultó nueva y estuvo constituida por 212 estudiantes. ${ }^{4}$

La pregunta 1, de tipo abierto, apuntaba fundamentalmente al MFDMet. La pregunta 2, por su parte, suponía la comprensión del MFCCHet y del MFDMet, y, finalmente, la pregunta 3 apelaba al conocimiento de elementos de los tres modelos ad hoc.

3. Las respuestas afirmativas fueron sistematizadas en caminos resolutivos organizando las palabras clave en secuencias. El análisis de la totalidad de los caminos resolutivos permitió la construcción de una red semántica poblacional. En un trabajo investigativo independiente (Garófalo et al., 2013) se efectúa el análisis comparativo con redes provenientes de las respuestas de otras poblaciones de estudiantes.

4. El análisis del cuestionario semiestructurado también fue poblacional, sin intención ni registro para analizar continuidades individuales a las respuestas de las entrevistas previas. 
Cuadro 3.

Cuestionario semiestructurado

1. Imagínate a dos personas en situaciones distintas: una que se acaba de levantar de dormir toda la noche, y la otra que terminó de comer hace dos horas. ¿Consideras que estar pasando por distintos momentos influye en el origen de la glucosa que se utiliza para la respiración celular? Justifica.

2. ¿Piensas que existe alguna relación entre los carbonos de la materia orgánica que forman parte, por ejemplo, de los fideos que podamos haber comido en un almuerzo y las moléculas de $\mathrm{CO}_{2}$ que se podrían eliminar al exhalar aire? Elige una de las siguientes opciones marcándola con una X:

a) No, porque el $\mathrm{CO}_{2}$ que se elimina al exhalar el aire es el mismo que ingresó al inhalar.

b) No, porque el $\mathrm{CO}_{2}$ que se elimina al exhalar el aire es el producto de la respiración aeróbica de la glucosa y no tiene nada que ver con lo que se comió.

c) No, porque el $\mathrm{CO}_{2}$ que se elimina al exhalar el aire proviene de la glucosa del glucógeno que tenemos de reserva y nunca de la alimentación.

d) Sí, porque el $\mathrm{CO}_{2}$ que se elimina al exhalar el aire proviene de la oxidación química de moléculas orgánicas que incorporamos con la alimentación.

e) Ninguna de las opciones anteriores.

f) Sí, porque el $\mathrm{CO}_{2}$ que se elimina al aire es el producto de la digestión de los alimentos, pero se elimina por el intestino como metano, como las heces.

g) No sé.

3. Una persona ingiere pan. ¿Dónde se podrían encontrar, luego de algunas horas, los carbonos de la glucosa que forman parte del almidón de ese pan? Elige una de las siguientes opciones marcándola con una X:

a) Se detectará en el aire, ya que parte de la glucosa se oxidó y se eliminó como $\mathrm{CO}_{2}$.

b) Se detectará en moléculas de glucógeno en hepatocitos (células del hígado).

c) Se detectará en adipocitos, formando parte de moléculas de triglicéridos.

d) Se detectará en distintas biomoléculas de las células de distintos tejidos.

e) Todas las opciones anteriores son correctas.

f) No se detectará en ninguna célula de ningún tejido de este individuo porque la glucosa se oxidó totalmente y se eliminó como $\mathrm{CO}_{2}$.

g) Se detectará en las heces del individuo y/o como metano.

b) Ninguna de las opciones anteriores es correcta.

i) No sé.

\section{RESULTADOS Y DISCUSIÓN}

\section{Entrevista}

Las respuestas de los estudiantes provenientes de las entrevistas arrojaron numerosas evidencias empíricas de fallos en los aprendizajes. ${ }^{5}$

El 66\% de los estudiantes indagados sostuvo, al responder al problema abierto, que no existe la posibilidad de encontrar en la atmósfera, en algún momento, alguno de los átomos de carbono que forman parte de la glucosa del almidón de la pizza. Esto significa que los conceptos inherentes al MFCCHet no han sido comprendidos por estos estudiantes, a pesar de que son generalmente enseñados desde la escuela secundaria, y que fueron particularmente intensificados en este curso universitario de biología.

5. Las evidencias empíricas deben considerarse como fotos del conocimiento declarativo de las poblaciones objeto de la indagación. Es decir, los porcentajes podrían cambiar de un cuatrimestre a otro, o de un año a otro, conforme cambian las poblaciones de estudiantes; por tanto, deben valorarse por el contenido que revelan -en cuanto a obstáculos de aprendizaje-, y no considerarse como cifras absolutamente reproducibles. 
El 34\% restante respondió afirmativamente al problema abierto. Sin embargo, llamó la atención que el $50 \%$ de estos estudiantes introdujera en sus respuestas expresiones tales como «la glucosa se dirige hacia las células que más la necesitan», y que un 18\% se refiriera a «la formación y eliminación de gas metano", expresiones que se reiteraron en las indagaciones subsiguientes.

A continuación se analizan las respuestas obtenidas a las tres preguntas de la entrevista. Los resultados se resumen en la tabla 1, discriminándose en forma separada las respuestas del grupo que contestó negativamente al problema abierto (Rneg) de las del grupo que lo hizo afirmativamente (Rafir).

Pregunta 1. ¿Sabes de dónde proviene la glucosa involucrada en la respiración celular?

El 85\% de los estudiantes que contestaron negativamente al problema abierto declaró explícitamente desconocer el origen de la glucosa involucrada en la respiración celular; el 10\% expresó que «la glucosa ya forma parte de las moléculas que hay en nuestras células», y el 5\% restante señaló la glucólisis como origen ${ }^{6}$ de la glucosa. Es decir, no hubo respuestas correctas en este grupo Rneg.

De los estudiantes que respondieron afirmativamente al problema abierto, el 36\% respondió de manera correcta que «la glucosa proviene de la dieta» o «de lo que comemos». Cabe mencionar que en esta asignatura no se describen las vías de obtención de glucosa como gluconeogénesis y glucogenolisis. No obstante, se señala el papel del glucógeno y que principalmente los hepatocitos pueden sintetizar glucosa a partir de precursores tales como aminoácidos, lactato o glicerol.

Un 10\% señaló explícitamente desconocer el origen de la glucosa involucrada en el proceso de respiración celular. Un 38\% respondió erróneamente que «la glucosa ya forma parte de las moléculas de nuestras células» y un 16\% que «la glucosa proviene de la glucólisis».

Pregunta 2. ¿Cómo y por dónde se incorpora la glucosa a la sangre?

No hubo respuestas correctas para el grupo Rneg. El 70\% de ellos expresó desconocer cómo se incorpora la glucosa a la sangre; el 25\% respondió que «ya forma parte de nuestras células», y el 5\% afirmó que «nuestras células la sintetizan».

El 45\% de los estudiantes Rafir contestó correctamente "por el intestino». El 21\% declaró "pero no sé cómo»; el 26\% sostuvo erróneamente que «la glucosa ya forma parte de nuestras células», y el 8\% señaló que «nuestras células sintetizan glucosa».

Pregunta 3. ¿Qué sucede con la glucosa una vez en sangre?

El 80\% de los estudiantes Rneg señaló desconocer qué sucede con la glucosa una vez en sangre, y el 20\% expresó que la «glucosa se dirige hacia las células que más la necesitan». Es decir, no hubo respuestas correctas en este grupo Rneg.

En el grupo de los estudiantes Rafir tampoco se registraron respuestas correctas; el 56\% manifestó explícitamente que desconocía la respuesta, y el 44\% expresó que la «glucosa se dirige hacia las células que más la necesitan».

6. Esta respuesta podría provenir del énfasis sobre el tema glucólisis que se hace en las clases de Biología; o bien podría haber surgido porque los estudiantes habrían interpretado que la pregunta apuntaba a cuál es el proceso bioquímico por el que comienza la respiración celular a partir de la glucosa.

7. Generalmente no se explican los posibles orígenes de la glucosa, sino que se hace especial énfasis en el papel energético de la molécula. Tal es el caso de los estudiantes que sólo relacionan la glucosa con su función energética (Oliveira et al., 2003). 
Tabla 1.

Porcentajes de respuestas de 182 estudiantes a las preguntas de la entrevista

\begin{tabular}{|l|c|c|}
\hline \multicolumn{2}{|c|}{ Pregunta 1. ¿Sabes de dónde proviene la glucosa involucrada en la respiración celular? } \\
\hline Respuestas correctas & Rneg (120 estudiantes, 66\%) & Rafir (62 estudiantes, 34\%) \\
\hline Manifiesta «desconocer» & $0 \%$ & $36 \%$ \\
\hline Argumenta que «forma parte de las células» & $85 \%$ & $10 \%$ \\
\hline Argumenta que «proviene de glucólisis» & $10 \%$ & $38 \%$ \\
\hline \multicolumn{1}{|c|}{ Pregunta 2. ¿Cómo y por dónde se incorpora la glucosa a la sangre? } \\
\hline Respuestas correctas & $0 \%$ & $16 \%$ \\
\hline Manifiesta «desconocer» & $70 \%$ & $45 \%$ \\
\hline Argumenta que «forma parte de las células» & $25 \%$ & $21 \%$ \\
\hline Argumenta que «nuestras células la sintetizan» & $5 \%$ & $26 \%$ \\
\hline \multicolumn{2}{|c|}{ Pregunta 3. ¿Qué sucede con la glucosa una vez en sangre? } \\
\hline Respuestas correctas & $0 \%$ & $8 \%$ \\
\hline Manifiesta «desconocer» & $80 \%$ & $0 \%$ \\
\hline Argumenta que «se dirige a donde más se necesita» & $20 \%$ & $56 \%$ \\
\hline
\end{tabular}

En resumen, surge claramente de esta tabla que del 66\% de los estudiantes constituyentes del grupo Rneg no construyó el modelo mental del MFCCHet, ni tampoco cuestiones básicas de los modelos MFDMet y MFCHGSang, evidenciado en los porcentajes de desconocimiento o de argumentaciones erróneas a las preguntas 1,2 y 3.

Por otro lado, los estudiantes que respondieron afirmativamente al problema abierto, pertenecientes al grupo Rafir, habrían comprendido el MFCCHet. Sin embargo, la pregunta 1 confirmó que solo un 36\% de este grupo de estudiantes comprendió este modelo.

Con relación al MFDMet, si bien el 36\% respondió correctamente la pregunta 1, el hecho de que ningún estudiante contestara la pregunta 3 indica una construcción parcial del MFDMet. Del mismo modo, que el $45 \%$ respondiera correctamente la pregunta 2 , pero que ninguno hubiera respondido la pregunta 3, indica una construcción parcial del MFCHGSang.

Estos resultados indicarían, en principio, que incluso los estudiantes que parecieron haber construido el MFCCHet a partir de responder afirmativamente al problema abierto manifestaron deficiencias cuando mostraron sus marcos de significación y argumentación frente a las otras preguntas de la entrevista.

\section{Cuestionario semiestructurado}

Como se mencionó anteriormente, el objetivo de aplicar un cuestionario semiestructurado tres semanas después de las entrevistas fue investigar si se confirmaban los fallos de aprendizaje detectados en la entrevista.

La pregunta 1 (cuadro 3) tiene estructura abierta; por tanto, permitió identificar categorías semánticas emergentes en las respuestas (Sirvent, 2003), resumidas en la tabla 2.

Un 13\% respondió «no sé». El 69\% contestó que el origen de la glucosa que se utiliza en la respiración celular no depende del estado nutricional por el que atraviesa un organismo. Las justificaciones fueron: el 31\% consideró que «durante la noche las células consumen el ATP de la glucosa que se ingirió durante el día»; el 17\% que «la glucosa es parte de moléculas que ya hay en nuestras células»; el 13\% afirmó que la glucosa «se sintetiza siempre y que no tiene nada que ver con el momento de ali- 
mentación por el que atraviesa la persona», y el 8\% sostuvo que «la glucosa proviene de la glucólisis». Estos resultados hacen inferir que estos estudiantes no han comprendido los elementos del MFDMet.

Solo un 18\% afirmó que el origen de la glucosa que se utiliza en la respiración celular depende del momento nutricional por el que atraviesa un organismo. Estos estudiantes han comprendido elementos básicos del MFDMet; sin embargo, en sus justificaciones no hicieron referencia a elementos del MFCHGSang, como por ejemplo los distintos modos en que el organismo mantiene la homeostasis de la glucosa en sangre (aplicados a la situación planteada en el problema). Muchos estudiantes señalaron desconocer qué procesos ocurrían en los períodos en los que no hay ingesta.

Tabla 2.

Respuestas de los estudiantes a la pregunta 1 del cuestionario semiestructurado (porcentajes calculados sobre 202 respuestas, dado que 10 estudiantes no respondieron esta pregunta)

\begin{tabular}{|c|c|c|c|c|c|c|}
\hline & $\begin{array}{l}\text { Manifiesta } \\
\text { desconocer la } \\
\text { respuesta } \\
\text { (26 estudian- }\end{array}$ & \multicolumn{4}{|c|}{$\begin{array}{c}\text { El origen de la glucosa no depende del estado nutricional del } \\
\text { organismo } \\
\text { (139 estudiantes, 69\%) }\end{array}$} & $\begin{array}{c}\text { El origen de la } \\
\text { glucosa depen- } \\
\text { de del estado } \\
\text { nutricional del } \\
\text { organismo (37 } \\
\text { estudiantes, } \\
18 \% \text { ) }\end{array}$ \\
\hline Argumentos & «No sé» & $\begin{array}{c}\text { «Durante la } \\
\text { noche las célu- } \\
\text { las consumen } \\
\text { el ATP de la } \\
\text { glucosa que se } \\
\text { ingirió durante } \\
\text { el día» } \\
(31 \%)\end{array}$ & $\begin{array}{l}\text { «La glucosa } \\
\text { es parte de } \\
\text { las moléculas } \\
\text { que ya hay } \\
\text { en nuestras } \\
\text { células» } \\
(17 \%)\end{array}$ & $\begin{array}{l}\text { «Se sintetiza } \\
\text { siempre y que } \\
\text { no tiene nada } \\
\text { que ver con } \\
\text { el momento } \\
\text { de alimenta- } \\
\text { ción por el } \\
\text { que atraviesa } \\
\text { la persona» } \\
(13 \%)\end{array}$ & $\begin{array}{l}\text { «La glucosa } \\
\text { proviene de la } \\
\text { glucólisis» } \\
(8 \%)\end{array}$ & $\begin{array}{l}\text { «La glucosa } \\
\text { siempre viene } \\
\text { de la dieta, } \\
\text { cuando no se } \\
\text { come no sé de } \\
\text { dónde» }\end{array}$ \\
\hline
\end{tabular}

La pregunta 2 (cuadro 3), de opción múltiple, indagaba si los estudiantes pensaban que existe alguna relación entre los carbonos de la materia orgánica del alimento y las moléculas de $\mathrm{CO}_{2}$ que se podrían exhalar al aire. Las opciones de respuestas $a), b), c), e$ ) o f) son incorrectas. La $d$ ) es la opción correcta. La opción $g$ ) permitía mostrar autoconciencia sobre la propia falta de conocimientos. Las respuestas (tabla 3) mostraron que la opción correcta d) fue elegida solo por un 13\% de estudiantes, que habrían comprendido los conceptos básicos del MFCCHet.

El 71\% del total eligió opciones incorrectas. La opción a), mayoritariamente elegida (32\%), evidencia que los estudiantes no lograron construir ninguno de los modelos ad hoc. Un $24 \%$ eligió la opción b); un $6 \%$ eligió la opción $c$ ) y un $16 \%$ eligió las opciones $e$ ) y g) (estos estudiantes no comprendieron el MFCCHet ni el MFDMet). El 9\% eligió la opción $f$ ) (estos estudiantes habrían construido una idea alternativa resistente al aprendizaje de los conceptos del MFCCHet).

Tabla 3.

Respuestas de 212 estudiantes a la pregunta 2 del cuestionario semiestructurado. La opción $d$ ) es la correcta

\begin{tabular}{|l|c|c|c|c|c|c|c|}
\hline Opciones de la Pregunta 2 & $a)$ & $b)$ & $c)$ & $d)$ & $e)$ & $f)$ & $g$ ) \\
\hline Porcentaje de elección & $32 \%$ & $24 \%$ & $6 \%$ & $13 \%$ & $5 \%$ & $9 \%$ & $11 \%$ \\
\hline
\end{tabular}


La pregunta 3 (cuadro 3) de opción múltiple indagaba específicamente sobre conocimientos acerca de los múltiples destinos de los átomos de carbono de la glucosa proveniente de la alimentación.

La elección de las opciones $a$ ), b), $c$ ), d) o f) confirmarían que el estudiante establecía relaciones parciales (fragmentadas) entre alimentación y las vías metabólicas activadas; la opción $e$ ) es la correcta; las respuestas $g$ ) y h), incorrectas.

Las respuestas (tabla 4) mostraron que la opción correcta $e$ ) fue elegida solo por el $4 \%$ de estudiantes.

El 33\% del total eligió opciones parcialmente correctas: la $a$ ) el 20\%; la $b$ ) el 5\%; la $c$ ) el 2\% y la d) fue elegida por el $6 \%$.

La opción $f$ incorrecta fue elegida por el $24 \%$ de estudiantes, que por consiguiente no habrían comprendido el MFCCHet ni el MFDMet.

La opción incorrecta $g$ ) fue elegida por el $26 \%$. Estos estudiantes habrían construido una idea alternativa resistente al aprendizaje de los conceptos del MFCCHet. Estas respuestas, en conjunto, refuerzan la información obtenida para el problema abierto de las entrevistas.

La opción i) fue elegida por el 14\% del total, y la opción h) no fue elegida por ningún estudiante. Ambos grupos de estudiantes no reconocen elementos de los MFCCHet ni el MFDMet.

Tabla 4.

Respuestas de 212 estudiantes a la pregunta 3

del cuestionario semiestructurado. La opción correcta es la $e$ )

\begin{tabular}{|l|c|c|c|c|c|c|c|c|c|}
\hline Opciones de la pregunta 3 & $a)$ & $b)$ & $c)$ & $d)$ & $e)$ & $f)$ & $g)$ & h) & i) \\
\hline Porcentaje de elección & $20 \%$ & $5 \%$ & $2 \%$ & $6 \%$ & $4 \%$ & $24 \%$ & $26 \%$ & $0 \%$ & $14 \%$ \\
\hline
\end{tabular}

\section{NUEVA PROPUESTA TEÓRICA: OBSTÁCULOS EPISTEMOLÓGICOS DE APRENDIZAJE DE TIPO BRECHA Y DE TIPO PUENTE}

El complejo discurso científico es fraccionado en secuencias para su enseñanza en clases, o para su escritura en capítulos de libros de texto (Adúriz-Bravo e Izquierdo, 2009). A su vez, la trama conceptual que interconecta esos fragmentos debe ser reconstruida por cada sujeto que aprende para organizar sus modelos mentales idiosincrásicos. Tales modelos mentales idiosincrásicos, idealmente, deberían ser similares a los modelos mentales expertos. Sin embargo, esta tarea no resulta sencilla a los estudiantes noveles, aún de nivel universitario.

Los fallos detectados en los aprendizajes de los estudiantes para el MHC, con relación a los tres modelos científicos definidos ad hoc, nos llevan a nuevas reflexiones teóricas. Proponemos categorizar dos tipos diferentes de obstáculos de aprendizaje: los de tipo brecha y los de tipo puente.

Obstáculo epistemológico de tipo brecha: Consiste en la falta de construcción de modelos mentales que funcionen como estructuras de conocimiento previamente formadas, tales que resulten organizadoras de los nuevos contenidos que deben ser aprendidos.

El sujeto que toma conciencia de su brecha cognitiva podría responder con un «No sé» frente a una pregunta temática, es decir, durante su búsqueda cognitivo-reflexiva el sujeto tomaría conciencia de que no ha construido un modelo mental funcional para encontrar una respuesta adecuada. Sin embargo, la actitud del sujeto podría ser diferente y responder mediante heurísticos, aunque este mecanismo generalmente conduce a errores (Christian y Talanquer, 2012).

Obstáculo epistemológico de tipo puente: Se origina en ideas cerradas y erróneas que funcionan como un modelo mental idiosincrásico -diferente del modelo mental del experto-, que da certezas a los sujetos sobre sus conocimientos acerca de un tema. Así, el obstáculo epistemológico tipo puente permite al sujeto contestar desde un modelo mental idiosincrásico funcional, pero, por este motivo, no se da cuenta de sus fallos en la construcción de un modelo mental experto adecuado. 
La interpretación de los resultados previamente informados, a partir de la nueva propuesta teórica de clasificación de obstáculos de aprendizaje permite concluir que:

a) En los estudiantes de la muestra existen numerosos obstáculos epistemológicos de tipo brecha que se corresponden con evidencias sobre la no-construcción o construcciones parciales de los tres modelos científicos ad hoc definidos en este trabajo: MFCCHet, MFCHGSang y MFDMet. Los estudiantes que nunca han comprendido estos modelos pudieron haber estudiado memorísticamente gran cantidad de datos sobre MHC, e incluso haber aprobado las evaluaciones tradicionales; sin embargo, cabe preguntarnos, como docentes, si estos aprendizajes aislados constituyen algún objetivo educativo. ${ }^{8}$

A partir de los instrumentos utilizados en esta indagación, puede decirse que la contestación «No sé» indica claramente obstáculos tipo brecha. Por ejemplo, en la entrevista (tabla 1) realizada a 182 estudiantes, la pregunta 1 evidenció este tipo de respuestas para un $85 \%$ de los Rneg y un $10 \%$ de los Rafir, lo que representa el $60 \%$ de la muestra total. Para la pregunta 2 se registraron un 70 y un $21 \%$, respectivamente, que representa un $53 \%$ de la muestra total, y análogamente, para la pregunta 3, el desconocimiento explícito por parte de los estudiantes representa un $72 \%$ del total.

Este tipo de obstáculo tipo brecha también se detectó en las respuestas al cuestionario semiestructurado (cuadro 3). Por ejemplo, el 13\% de los estudiantes manifestó desconocer la respuesta a la pregunta 1 (tabla 2), y el 18\% que aseguró desconocer el origen de la glucosa utilizada en la respiración celular cuando esta no es ingerida. Otros ejemplos provienen del $11 \%$ de las respuestas a la pregunta 2 (tabla 3), y del $14 \%$ a la pregunta 3 (tabla 4 ). Asimismo, este instrumento de indagación permitió detectar mediante la pregunta 2, de opción múltiple, otros posibles obstáculos de tipo brecha, como por ejemplo la elección de opciones $a$ ), b), c), d) y f), que daban respuestas parciales a la pregunta 3 (tabla 4).

b) A partir de los instrumentos utilizados en la indagación pueden distinguirse obstáculos epistemológicos de tipo puente que son básicamente argumentos que permiten a los estudiantes brindar explicaciones mediante modelos mentales idiosincrásicos. Por ejemplo, las afirmaciones de «la glucosa que se utiliza en la respiración ya forma parte de nuestras células», o «la glucosa que se utiliza en la respiración celular la sintetizan nuestras células», que se corresponden entre un 10 a un $30 \%$ del grupo Rneg (tabla 1, preguntas 1 y 2, respectivamente). Este obstáculo también se detectó en un 38 y 34\% del grupo Rafir (tabla 1, preguntas 1 y 2, respectivamente). Ambas expresiones evidenciarían un modelo mental idiosincrásico subyacente por el cual la glucosa no necesita ser incorporada por alimentos, ni ser sintetizada por el organismo, en caso de falta de ingesta. Este obstáculo impediría la construcción del MFCCHet y del MFDMet, por cuanto asume que el suministro de la glucosa que se involucra en la respiración celular sería siempre endógeno e inagotable. Especulamos que este tipo de concepción provendría de interpretaciones erróneas sobre aspectos del discurso de los modelos científicos explícitos en los que, por ejemplo, el docente o el texto presentan el proceso de glucólisis diciendo «una molécula de glucosa», sin una reflexión fisiológica ni conceptual sobre el significado estequiométrico de las reacciones involucradas.

Un segundo obstáculo epistemológico tipo puente fue encontrado durante la entrevista, y se corresponde con el 20\% de los estudiantes del grupo Rneg y el 44\% de los Rafir, que sostuvieron

8. Son frecuentes los contenidos de enseñanza de MHC llenos de nombres de compuestos y enzimas involucrados en las rutas metabólicas, así como de ejercicios de conteo de moléculas de ATP, según metabolismos secuenciales o cíclicos. Estos contenidos son generalmente evaluados, y por tanto aprendidos por los estudiantes para aprobar los exámenes. 
que «la glucosa se distribuye hacia donde más se necesita» (tabla 1, pregunta 3). Esta afirmación está relacionada con un modelo mental idiosincrásico que otorga intencionalidad ${ }^{9}$ a los procesos metabólicos o a las moléculas, y que impediría siquiera plantearse los conceptos fundamentales del MFCHGSang.

Otros obstáculos epistemológicos de tipo puente fueron detectados en las opciones elegidas como respuestas a la pregunta 1 del cuestionario semiestructurado (cuadro 3). Por ejemplo, las diferentes argumentaciones que sostuvo el 69\% de los estudiantes para justificar que el origen de la glucosa no depende del estado nutricional del organismo (tabla 2).

En la pregunta 2 de dicho cuestionario se detectó este tipo de obstáculos en el 76\% de los estudiantes que eligieron las opciones incorrectas (tabla 3).

Particularmente, el argumento del 26\% de los estudiantes, que afirmaron que «los carbonos del alimento solo pueden ser eliminados mediante la actividad bacteriana sobre los desechos de la materia orgánica producto del proceso digestivo, o por las heces, como metano» (pregunta 3 del cuestionario, tabla 4), constituye un obstáculo tipo puente que indica la construcción de un modelo mental idiosincrásico alternativo al del MFCCHet. Este modelo mental idiosincrásico quizás fuera originado en dos informaciones muy trabajadas en la escuela secundaria: la formación de biogás (metano), a partir de descomposición de materia orgánica, y la contribución al efecto invernadero del gas metano, proveniente del desecho gaseoso de los procesos digestivos del ganado (Schultz, 1996; Sánchez et al., 2001; INTA, 2010).

\section{CONSIDERACIONES FINALES}

Haber analizado errores de aprendizaje en función de los modelos mentales idiosincrásicos construidos por los estudiantes, alejados de los modelos mentales expertos, esperados para el aprendizaje del MHC, ha permitido generar una propuesta teórica que discrimina obstáculos epistemológicos subyacentes al aprendizaje. La presente categorización en obstáculos de tipo brecha y puente es un aporte innovador que permite una modalidad de análisis que va más allá de la simple enumeración de los errores de los estudiantes y que podría aplicarse a otras temáticas.

A partir de la gran cantidad de obstáculos epistemológicos detectados en este trabajo, cabe reflexionar sobre los criterios de selección de contenidos y sobre las metodologías de enseñanza aplicadas en los diferentes niveles educativos acerca del tema MHC. Asimismo, debería cuestionarse el sentido de forzar a los estudiantes a un aprendizaje centrado en el esfuerzo memorístico cuando, por ser novatos en estos temas, carecen de modelos mentales abarcadores que funcionen como conocimientos previos sobre los cuales engarzar comprensiva y sustentablemente los nuevos contenidos (Galagovsky, 2004a y b).

\section{AGRADECIMIENTOS}

Esta investigación fue financiada mediante los subsidios de la Universidad de Buenos Aires UBACyT X181 y UBACyT U011. S. J. G. realizó este trabajo como becaria doctoral de la Universidad de Buenos Aires.

9. La utilización de analogías antropomórficas (Gentner et al., 2001; Anissimov, 2003) es un recurso incluso didáctico, muy generalizado y particularmente utilizado por docentes y por científicos en sus razonamientos y explicaciones coloquiales (Dunbar, 2000). 


\section{REFERENCIAS BIBLIOGRÁFICAS}

Adúriz-Bravo, A. (2010). Concepto de modelo científico: Una mirada epistemológica de su evolución. En L. Galagovsky (coord.). Didáctica de las ciencias naturales: El caso de los modelos científicos. Buenos Aires: Lugar Editorial, pp. 141-161.

Adúriz-Bravo, A. y IzQuierdo, M. (2009). Un modelo de modelo científico para la enseñanza de las ciencias naturales. Revista Electrónica de Investigación en Educación en Ciencias, Año 4, Número especial 1, pp. 40-49.

Alberts, B.; Johnson A.; Lewis, J.; Raff, M.; Roberts, K. y Walter, P. (2002). Biología Molecular de la Célula (4.a ed.). Barcelona: Omega.

Anissimov, M. (2003). A Concise Introduction to Heuristics and Biases. Disponible en línea: http:// www.acceleratingfuture.com/michael/works/heuristicsandbiases.htm. (Última consulta, 10 de octubre de 2009).

Galagovsky, L. (2004a). Del aprendizaje significativo al aprendizaje sustentable. Parte 1: el modelo teórico. Enseñanza de las Ciencias, 22 (2), pp. 230-240.

Galagovsky, L. (2004b). Del aprendizaje significativo al aprendizaje sustentable. Parte 2: derivaciones comunicacionales y didácticas. Enseñanza de las Ciencias, 22 (3), pp. 349-364.

Galagovsky, L.; Di Giacomo, M.A. y Castelo, V. (2009). Modelos vs. dibujos: el caso de la enseñanza de fuerzas intermoleculares. Revista Electrónica de Enseñanza de las Ciencias, 8 (1), pp. 1-22. Disponible en línea: http://reec.uvigo.es/volumenes/volumen8/ART1_Vol8_N1.pdf. (Última consulta, 3 de abril de 2010).

Garófalo, S.J. (2010). Obstáculos epistémicos de aprendizaje del tema metabolismo de Hidratos de Carbono. Un estudio transversal. Tesis doctoral, Facultad de Ciencias Exactas y Naturales, Departamento de Química Biológica, Universidad de Buenos Aires.

Garófalo, S.J.; Alonso, M. y Galagovsky, L. (2013). Redes semánticas poblacionales: un instrumento metodológico para la investigación educativa. Ciência \& Educação (Bauru) (enviado).

Banet Hernández, E. (2001). Los procesos de la nutrición humana. Colección Didáctica de las Ciencias Experimentales. Madrid: Síntesis Educación.

Banting, F.G.; Best, C.H.; Collip, J.B.; Macleod, J.J.R. y Noble, E.C. (1922). The Effect of Pancreatic Extract (Insulin) on normal rabbits, Amer. J. Physiol., 62, pp. 162-176.

Berg, J.M.; Tymoczko, J.L. y Stryer, L. (2007). Bioquimica. (6. a ed.). Barcelona: Reverté.

Díaz Hernández, D.P. y Burgos Herrera, L.C. (2002). ¿Cómo se transporta la glucosa a través de la membrana celular? Iatreia, 15 (3) pp. 179-189.

Campbell, N. y Reece, J.B. (2005). Biología (7. a ed.). Madrid: Médica Panamericana, 2007.

Christian K. y Talanquer V. (2012). Modes of reasoning in self-initiated study groups in chemistry. Chemistry Education Research and Practice, 13 (3), pp. 286-295.

http://dx.doi.org/10.1039/c2rp20010d

Cliff, W.H. (2006). Case study and the remediation of misconceptions about respiratory physiology. Adv Physiol Educ, vol. 30, pp. 215-223.

http://dx.doi.org/10.1152/advan.00002.2006

Curtis, H.; Barnes, N.S.; Schnek, A. y Massarini, A. (2008). Biología (7. a ed.). Buenos Aires: Médica Panamericana.

Díaz Hernández, D.P. y Burgos Herrera, L.C. (2002). ¿Cómo se transporta la glucosa a través de la membrana celular? Iatreia, 15 (3), pp. 179-189.

Dunbar, K. (2000). How scientists think in the real world: implication for science education. Journal of Applied Developmental Psychology, 21(1), pp. 49-48.

http://dx.doi.org/10.1016/S0193-3973(99)00050-7 
Ericsson, K.A. y Simon, H.A. (1999). Protocol Analysis: Verbal Reports as Data. Cambridge, MA: MIT Press.

Estany, A. (1993). Introducción a la filosofía de la ciencia. Barcelona: Crítica.

Gagliardi, R. (1986). Los conceptos estructurantes en el aprendizaje por investigación. Enseñanza de las Ciencias, 4 (1), pp. 30-35.

Gentner, D.; Holyonk, K. y Kokinov, B. (2001). The analogical mind. Perspective from cognitive science. Cambridge, MA: MIT Press.

Gutiérrez, R. (2005). Polisemia actual del concepto «modelo mental»: Consecuencias para la investigación didáctica. Investigaçóes em Ensino de Ciências, 10(2), pp. 209-226. Disponible en línea: http://www.if.ufrgs.br/public/ensino/vol10/n2/v10_n2_a4.htm.

Hudson, J. (1992). The History of Chemistry. New York: Chapmann \& Hall. http://dx.doi.org/10.1007/978-1-4684-6441-2

Instituto Nacional de Tecnología Agropecuaria (2010). Buenos Aires: INTA. Disponible en línea: http://www.inta.gov.ar/info/bioenergia/Manual\%20para\%20la\%20producci\%C3\%B3n\%20 de\%20biog\%C3\%A1s\%20del\%20IIR.pdf. (Última consulta, 20 de julio de 2010).

Johnson-Laird, P.N. (2005). Mental models in thought. En K. Holyoak y R.J. Sternberg (eds.). The Cambridge Handbook of Thinking and Reasoning. Cambridge: Cambridge University Press.

Justi, R. (2006). La enseñanza de las ciencias basada en la elaboración de modelos. Enseñanza de las Ciencias, 24 (2), pp. 173-184.

Lehninger, A. (1978). Bioquimica (Trad. F. Calvet Prats y J. Bozal Fes) (2. ${ }^{a}$ ed.). Barcelona: Omega.

Lombardi, O. (2010). Los modelos como mediadores entre teoría y realidad. En L. Galagovsky (coord.). Didáctica de las ciencias naturales: El caso de los modelos cientificos. Buenos Aires: Lugar Editorial, pp. 83-93.

Luz, M.R.P. (2008). Glucose as the sole metabolic fuel: a study on the possible influence of teachers' knowledge on the establishment of a misconception among Brazilian high school students. $A d v$. Physiol. Educ., 32, pp. 225-230. http://dx.doi.org/10.1152/advan.00050.2007

Luz, M.R.P.; Aguiar De Oliveira, G.; Ribeiro De Sousa, C. y Da Poian, A.T. (2008). Glucose as the Sole Metabolic Fuel: The Possible Influence of Formal Teaching on the Establishment of a Misconception About Energy-yielding Metabolism Among Students from Rio de Janeiro, Brazil. Biochem. Mol. Biol. Educ., 36 (6), pp. 407-416. http://dx.doi.org/10.1002/bmb.20235

Moreira, M.A.; Greca, M.I. y Rodríguez Palmero, M.L. (2002). Modelos mentales y modelos conceptuales en la Enseñanza \& aprendizaje de las ciencias. Revista Brasileira de Investigação em Educação em Ciências, 2 (3), pp. 36-56.

Oliveira, G.A.; Sousa, C.R.; Da Poian, A.T. y Luz, M.R.P. (2003). Students' misconception about energy-yielding metabolism: Glucose as the sole metabolic fuel. Adv. Physiol. Educ. 27, pp. 97-101. http://dx.doi.org/10.1152/advan.00009.2003

Palmer, G. (2001). Biosciences 302, Course notes. Houston: Rice University. Disponible en línea: http://www.bioc.rice.edu/-graham/Bios302/. (Última consulta, 1 de septiembre de 2010).

Passos, R.; SÉ, A.; Wolff, V.; Nobrega, Y.K.M. y Hermes-Lima, M. (2006). Pizza and pasta help students learn metabolism. Adv. Physiol. Educ., 30, pp. 89-93.

http://dx.doi.org/10.1152/advan.00044.2005

Pogozelski, W.; Arpaia, N. y Priore, S. (2005). The Metabolic Effects of Low-carbohydrate Diets and Incorporation into a Biochemistry Course. Biochem. Mol. Biol. Educ., 33 (2), pp. 91-100. http://dx.doi.org/10.1002/bmb.2005.494033022445 
Ross, P.M.; Tronson, D.A. y Ritchie, R.J. (2008). Glycolysis \& the Krebs Cycle using role-play. The American biology teacher, 70 (3), pp. 163-168.

http://dx.doi.org/10.1662/0002-7685(2008)70[163:ICUOGT]2.0.CO;2

SÁnchez, E.; Weiland, P. y Martin, A. (2001). Effect of substrate concentration and temperature on the anaerobic digestion of piggery waste in a tropical climate. Process Biochemistry, 37, pp. 483-489. http://dx.doi.org/10.1016/S0032-9592(01)00240-0

Schultz, H. (1996). Biogas-Praxis. Freiburg: Ökobuch.

Sirvent, M.T. (2003). Cuadro Comparativo entre Lógicas según dimensiones del Diseño de Investigación. En El Proceso de Investigación. Investigación y Estadística I (2.a ed.). Buenos Aires: Cuadernos de la Oficina de Publicaciones de la Facultad de Filosofía y Letras (Opfyl).

Songer, C. y Mintzes, J. (1994). Understanding Cellular Respiration: An analysis of conceptual changes in college Biology. Journal of Research in Science Teaching, 31(6), pp. 621-637. http://dx.doi.org/10.1002/tea.3660310605

Teixidó Gómez, F. (2003). Historia de la ciencia española en 25 estampas. León: Lancia ediciones. 


\title{
New theoretical proposal on epistemological learning obstacles. The case of carbohydrate metabolism
}

\author{
Sofía J. Garófalo, Manuel Alonso \\ Departamento de Ciencias Biológicas, Ciclo Básico Común, \\ Universidad de Buenos Aires, Ramos Mejía 84I, CI405CAE, Ciudad de Buenos Aires, Argentina \\ sjgarofalo@gmail.com,m_alonso@live.com.ar \\ Lydia R. Galagovsky \\ Facultad de Ciencias Exactas y Naturales, Instituto Centro de Formación e Investigación en Enseñanza de las Ciencias (CEFIEC), \\ Universidad de Buenos Aires, Ramos Mejía 84I, CI405CAE, Ciudad de Buenos Aires, Argentina \\ lyrgala@qo.fcen.uba.ar
}

The use of three ad hoc scientific models on the metabolism of carbohydrates allows us to identify the many learning errors made by university students.

The three models are as follows:

Physiological model of the carbon cycle in Heterotrophs: Understanding the redox processes of carbon compounds. These processes involve organic and inorganic chemical species of carbon compounds and are present both in the atmosphere and in autotrophic and heterotrophic organisms.

Physiological model on the availability of metabolites: Understanding the existence of small molecules as a core part of all metabolisms, and that biomolecules are constructed from a small number of precursors that connect catabolic and anabolic pathways.

Physiological model on the uptake and homeostasis of blood glucose: Understanding how the human body uptakes glucose from food and is homogeneously distributed, but how the uptake of glucose happens in a differential way and is done by the cells in the body tissues.

An oral survey involving an open problem and three questions was made to a sample of 182 volunteer students attending the first year of Biology at the University of Buenos Aires. Students had already been taught and assessed on topics concerning carbohydrate metabolism. Nevertheless, they still made important mistakes.

To confirm those results, a written questionnaire was given to students two weeks later. This time, the randomly-chosen population was of about 202 students.

An analysis of all results suggests the existence of underlying epistemological obstacles that hinder student understanding of the deep conceptual meaning of those defined scientific models.

A new theoretical approach helps interpret students' failures when considering two different epistemological learning obstacles (ELO): "Gap-type" and "Bridge-type" obstacles.

Gap-type ELOs correspond to cognitive obstacles arising from a lack of knowledge. This means that students' failure lies on the missing of previous mental models to which new abstract information can be connected to. Most of the answers stating "I don't know" are an example of these gap-type obstacles.

Bridge-type ELOs correspond to consolidated previous ideas that act as wrong alternative underlying mental models that hinder a right connection between new and old information already stored in students' minds. Examples of mistakes arising from this type of obstacles are the answers stating that "glucose used in respiration is already part of our cells and is not related to the human diet".

This theoretical contribution to the classification of learning obstacles based on proof allows an analysis that goes beyond the simple listing of students' misunderstandings.

Keywords: Learning difficulties; epistemological obstacles; metabolism; carbohydrates. 
